brought into different positions to get rid of spurious errors due to tilt. It is important to have each pivot tested as well by a Krupp microtest gauge which gives an accuracy to about $I \mu$. The instability of the frame carrying the moving wire has been completely removed now. In the discussion it was put forward that pivot errors and pivot flexure are to be taken into account.

A method of getting more independent bisections with the automatic travelling wire was described. The wire is purposely brought off the star and put on it again after a contact has come through. It was remarked that it is better to have a greater field over which the stars are followed than to have a great number of contacts per revolution. Anomalies will then be better eliminated. The Greenwich instrument has a glass circle. The circle is about $I$ inch thick and is 28 inches in diameter. The divisions are very clear. Care has been taken to compensate for extension due to temperature changes.

It was finally remarked that changes in the collimator with temperature may find their origin in different housing of collimators and meridian instrument.

Dr Moreau described the new meridian circle at Uccle. There is one circle of platinum-palladium, which is a very fine one; the other circle of "monelle", an alloy of iron, nickel and copper, has however many irregularities on its surface and has to be cleaned regularly. The reading of the microscopes is done photographically as inaugurated at Bergedorf. The pointings at the instrument micrometer are recorded by printing. There is no special advantage in having all the microscope photographs on the same film.

An account was given of a trial at the Askania Works to have the observations with the nadir basin supplemented by observations with a zenith mirror, the telescope being directed towards the zenith in the latter case. The mirror is floating in a ring-shaped mercury basin. In order to refer the measurements to the true horizon the measurements have to be done twice with the mirror turned $180^{\circ}$. The accuracy of the results so far obtained is not completely satisfactory as one does not yet come under one second of arc.

\title{
COMMISSION 9 (INSTRUMENTS)
}

\section{Acting President: Dr A. Couder. Secretary: Prof. H. Chrétien.}

La Commission s'est réunie le 5 août I938, à II heures.

La Commission examine en premier lieu la proposition de Mme Ed. Chandon suggérant qu'une enquête soit ouverte auprès des spécialistes des diverses disciplines astronomiques, sur les qualités exigées de l'œil, sur leurs mesures et leurs tolérances.

Les membres présents sont d'avis de demander à Mme Chandon de développer ses propositions, lesquelles seraient soumises, par voie de circulaire, aux membres de la Commission.

M. J. Svoboda donne des explications détaillées sur les déterminations d'équation personnelle dans les observations de passages dont il a été question dans le rapport préliminaire; il indique que l'erreur personnelle diminue quand la vitesse croît, et d'autre part qu'elle dépend, dans une certaine mesure, de la magnitude des étoiles et de leur coloration. 
M. F. Link expose le principe d'un spectrographe à prisme-objectif muni d'un dispositif photométrique. Les rayons venant de l'étoile traversent d'abord un prisme à vision directe, tombent ensuite sur l'objectif qui forme l'image du spectre sur la plaque. Le spectre est élargi à l'aide d'une lentille cylindrique placée devant la plaque. A côté de ce spectre central se forme une série de spectres de comparaison produits par un collimateur auxiliaire (et introduits dans l'appareil par réflexion sur une lame légèrement prismatique), dont la fente est couverte d'un filtre à échelons. Le prisme ne couvre qu'une fraction de la surface de l'objectif; la surface restée libre produit une image non dispersée dans le prolongement du spectre. Cette image sert de repère pour la détermination des longueurs d'onde. La fente du collimateur est éclairée par une lampe électrique maintenue à résistance constante par la méthode de M. G. Ribaud modifiée pour les mesures en campagne par M. Link. De plus la fente reçoit un faible flux d'une lampe à néon qui donne quelques raies spectrales fixant l'échelle de dispersion. Ainsi les spectres de comparaison et le spectre de l'étóle se forment simultanément dans des conditions aussi identiques que possible.

M. H. Brück rend compte des expériences actuellement faites à l'Observatoire de Physique Solaire de Cambridge, pour déterminer, au moyen d'un photomètre photoélectrique, le contour des lignes de Fraunhofer dans la région bleue du spectre solaire.

La méthode photoélectrique étant appliquée principalement pour servir de contrôle indépendant à un travail photographique antérieur, on a employé ici le même spectrographe à haute dispersion qui avait servi aux mesures précises des contours faites par le Dr Redman.

Comme dans le photomètre photoélectrique décrit par le Dr Dunham, la lumière provenant du spectrographe tombe sur une cellule et le courant photoélectrique amplifié est mesuré au moyen d'un galvanomètre. Des expériences ont montré la nécessité d'un amplificateur stable de haute sensibilité. On a adopté le montage de l'amplificateur à courant continu du Dr Bridge. On construit actuellement un appareil dans lequel une cellule à potassium dans le vide et un électromètre à valve triode relié à une grille formant résistance à fuite d'environ Io $^{11} \mathrm{ohms}$ forment les parties principales, la cellule et la valve avec fuite étant employées dans une enveloppe à vide. Les instabilités atmosphériques seront éliminées par un moyen qui permettra de vérifier de temps en temps l'intensité en un point quelconque du spectre continu au voisinage de la ligne étudiée, pendant l'enregistrement de son contour sur un cylindre tournant.

M. le Prof. R. Schorr donne des indications pratiques sur le moyen de donner aux plaques photographiques la courbure qui est nécessaire pour utiliser toute l'étendue du champ du télescope de Bernard Schmidt. M. Schorr présente à la Commission un chassis dans lequel les plaques ordinaires prennent la convexité requise par simple compression entre un appui antérieur annulaire et un appui dorsal pressant sur toute la surface de la plaque.

Des informations intéressantes sont apportées par M. M. L. Humason sur l'état d'avancement des travaux de l'Observatoire du Mont Palomar.

M. F. Moreau rend compte des expériences qui ont été faites, pour les besoins de l'Observatoire de Bruxelles, en vue du choix du métal le plus convenable à recevoir les graduations des cercles.

M. Nechvíle s'est occupé de la théorie du télescope aplanétique Ritchey-Chrétien. En partant des développements en séries donnés par $\mathbf{M}$. H. Chrétien, il a étudié pour ce système optique l'aberration longitudinale d'un rayon issu du centre de courbure du sommet du premier miroir et réfléchi sur lui seulement. L'expression analytique de cette aberration, dont la valeur est importante pour les recherches sur la qualité 
du miroir et pour sa construction, est donnée par un développement en série selon les puissances de la hauteur d'incidence du rayon.

M. F. Link signale l'utilité que présenterait un recueil de données numériques relatives aux propriétés des liquides susceptibles d'être employés comme milieux dispersifs. Il veut bien se charger de préparer un tel travail, ce dont la Commission le remercie vivement.

M. Henri Chrétien dépose sur le bureau, de la part du Capitaine Hellweg, de l'Observatoire Naval de Washington, quelques photographies du télescope RitcheyChrétien d'un mètre d'ouverture, qui vient d'être mis en service, ainsi que des photographies stellaires qui montrent l'efficacité du nouvel instrument.

M. A. Couder propose à la Commission, qui l'adopte à l'unanimité des membres présents, le projet de résolution suivant:

"Les auteurs d'instruments destinés à des recherches astronomiques, doués de propriétés optiques nouvelles, sont priés de donner toujours l'indication complète des données de construction, sous une forme qui permette un examen critique et éventuellement la reproduction: indices et dispersion des matières employées, courbures des surfaces, épaisseurs et distance des lentilles et, si possible, la description des aberrations résiduelles et des propriétés photométriques."

\title{
COMMISSION Io (SUNSPOTS AND CHARACTER FIGURES)
}

\author{
President: Prof. W. Brunner. \\ SECRETARY: Rev. LuIS RodÉs, S.J.
}

The Commission met on Thursday, August 4. The suggestion made by the President and by d'Azambuja to change the title of the Bulletin was first considered. After a short discussion on the most appropriate terms for this, a resolution was passed adopting Quarterly Bulletin on Solar Activity (Bulletin Trimestriel relatif à l'Activité Solaire). The President then referred to the advisability of continuing the publication of the character figures, separately for the whole disk and for the central zone, and proposed, seconded by Abettiand d'Azambuja, to omit in future the former and to retain only the latter, as long as they might be considered of some utility for statistical purposes. After a short exchange of views between several members (Abetti, d'Azambuja, Stratton, Royds, Rodés) who favoured the President's suggestion, a resolution was passed adopting it.

The President asked that the Commission support the Zurich Observatory when organizing a scheme of taking direct photographs of the Sun at short intervals, studying the manner of development of spots and spot groups. Attention was drawn to the fact that, since the character figures for the central zone are to be omitted in the Bulletin, there will be space for other data, which might be more useful. The President suggested publishing a time-diagram showing for each day the hours on which the Sun was under observation by the various spectrohelioscopes; he referred also to a suggestion of S. B. Nicholson to introduce daily character figures for bright chromospheric eruptions based on the number and intensity, and on the number of hours the Sun was observed per day. D'Azambuja finds that while some observers give the exact time during which they were actually observing the Sun, others merely give a total period of observation during which there might have been interruptions; he thought that gaps in the observations exceeding five minutes should 